\title{
Neuraminidase inhibitors, superinfection and corticosteroids affect survival of influenza patients
}

\author{
Nelson Lee ${ }^{1,2}$, Yee-Sin Leo ${ }^{3}$, Bin Cao ${ }^{4}$, Paul K.S. Chan ${ }^{2,5}$, W.M. Kyaw ${ }^{3}$, \\ Timothy M. Uyeki ${ }^{6}$, Wilson W.S. Tam ${ }^{7}$, Catherine S.K. Cheung ${ }^{1}$, Irene M.H. Yung ${ }^{1}$, \\ Hui Li ${ }^{4}$, Li Gu${ }^{4}$, Yingmei Liư ${ }^{4}$, Zhenjia Liư ${ }^{4}$, Jiuxin $\mathrm{Qu}^{4}$ and David S.C. Hui ${ }^{1,2}$

\begin{abstract}
Affiliations: ${ }^{1}$ Dept of Medicine and Therapeutics, The Chinese University of Hong Kong, Hong Kong, China. ${ }^{2}$ Stanley Ho Centre for Emerging Infectious Diseases, The Chinese University of Hong Kong, Hong Kong, China. ${ }^{3}$ Communicable Diseases Centre, Institute of Infectious Diseases and Epidemiology, Tan Tock Seng Hospital, Yong Loo Lin School of Medicine, National University of Singapore, Singapore, Singapore. ${ }^{4}$ Dept of Infectious Diseases and Clinical Microbiology, Beijing Chao-Yang Hospital, Beijing Institute of Respiratory Medicine, Capital Medical University, Beijing, China. ${ }^{5}$ Dept of Microbiology, The Chinese University of Hong Kong. Hong Kong. China. 'Influenza Division, National Center for Immunization and Respiratory Diseases, Centers for Disease Control and Prevention, Atlanta, GA, USA. ${ }^{7}$ School of Public Health and Primary Care, The Chinese University of Hong Kong, Hong Kong, China.
\end{abstract}

Correspondence: Nelson Lee, Division of Infectious Diseases, Dept of Medicine and Therapeutics, Faculty of Medicine, The Chinese University of Hong Kong, 9/F, Lui Che Woo Clinical Sciences Building, Prince of Wales Hospital, Shatin, Hong Kong SAR, China. E-mail: leelsndacuhk.edu.hk

ABSTRACT We aimed to study factors influencing outcomes of adults hospitalised for seasonal and pandemic influenza.

Individual-patient data from three Asian cohorts (Hong Kong, Singapore and Beijing; N=2649) were analysed. Adults hospitalised for laboratory-confirmed influenza (prospectively diagnosed) during 20082011 were studied. The primary outcome measure was 30-day survival. Multivariate Cox regression models (time-fixed and time-dependent) were used.

Patients had high morbidity (respiratory/nonrespiratory complications in $68.4 \%$, respiratory failure in $48.6 \%$, pneumonia in $40.8 \%$ and bacterial superinfections in $10.8 \%$ ) and mortality $(5.9 \%$ at 30 days and $6.9 \%$ at 60 days). $75.2 \%$ received neuraminidase inhibitors (NAI) $(73.8 \%$ received oseltamivir and $1.4 \%$ received peramivir/zanamivir; $44.5 \%$ of patients received NAI $\leqslant 2$ days and $65.5 \% \leqslant 5$ days after onset of illness); $23.1 \%$ received systemic corticosteroids. There were fewer deaths among NAI-treated patients ( $5.3 \%$ versus $7.6 \% ; \mathrm{p}=0.032$ ). NAI treatment was independently associated with survival (adjusted hazard ratio (HR) 0.28 , 95\% CI 0.19-0.43), adjusted for treatment-propensity score and patient characteristics. Superinfections increased (adjusted HR 2.18, 95\% CI 1.52-3.11) and chronic statin use decreased (adjusted HR 0.44, 95\% CI 0.23-0.84) death risks. Best survival was shown when treatment started within $\leqslant 2$ days (adjusted HR 0.20, 95\% CI 0.12-0.32), but there was benefit with treatment within 3-5 days (adjusted HR 0.35 , 95\% CI 0.21-0.58). Time-dependent analysis showed consistent results of NAI treatment (adjusted HR 0.39, 95\% CI 0.27-0.57). Corticosteroids increased superinfection (9.7\% versus $2.7 \%$ ) and deaths when controlled for indications (adjusted HR 1.73, 95\% CI 1.14-2.62). Early NAI treatment was associated with shorter length of stay in a subanalysis.

NAI treatment may improve survival of hospitalised influenza patients; benefit is greatest from, but not limited to, treatment started within 2 days of illness. Superinfections and corticosteroids increase mortality. Antiviral and non-antiviral management strategies should be considered.

@ERSpublications

NAI treatment, secondary infections and corticosteroids may impact on survival of hospitalised influenza patients http://ow.ly/ErOsT 


\section{Introduction}

Influenza virus infections cause excessive hospitalisations and deaths of adults during seasonal peaks and pandemics. While predominantly older individuals are hospitalised for infections caused by seasonal influenza virus strains, younger, previously healthy adults may be hospitalised for H1N1pdm09 infections; at present, co-circulation of these viruses occurs worldwide [1]. Data from Asia are relatively limited but the estimated influenza disease burden is at least similar to that in western countries [2-4]. Adults hospitalised with influenza may suffer from a wide range of complications including pneumonia, respiratory failure, multiorgan dysfunction, secondary infections and exacerbation of underlying conditions, resulting in high mortality $[3,5,6]$. However, the most optimal management approach for these patients with complicated influenza has remained unclear, as data from randomised, placebo-controlled trials are lacking [3]. Many observational studies performed during the 2009 pandemic reported effectiveness of neuraminidase inhibitors (NAI) started within 2 days of illness, but most were limited by a small sample size, single-centre design and lack of adjustment for confounders; benefits of later treatment appeared inconsistent [7]. Besides antiviral agents, the relative impacts of other modifiable disease conditions and the use of concomitant medications on clinical outcomes are uncertain. In particular, bacterial superinfection has been suggested to play a major role in influenza-related deaths $[3,5]$, and corticosteroids may sometimes be administered in an attempt to improve oxygenation in severe respiratory failure [8]. In this study, we aimed to determine clinical factors that affect survival and illness duration in adult patients hospitalised for influenza. We conducted a large, multicentre cohort study in three cities in Asia; individual-patient data of laboratory-confirmed seasonal or pandemic influenza virus infections were analysed in multivariate models. Such findings may optimise the overall management approach for severe influenza, leading to better clinical outcomes.

\section{Methods}

Study design

A pooled analysis of individual-patient data from three Asian influenza cohorts (Hong Kong, Singapore and Beijing) was performed [5, 6, 9-12]. Adults aged $>17$ years hospitalised for laboratory-confirmed influenza A or B virus infections from January, 2008 to December, 2011 (four calendar years) were included for analysis. Prince of Wales Hospital and Alice Ho Miu Ling Nethersole Hospital (Hong Kong), Tan Tock Seng Hospital (Singapore) and Beijing Chao-Yang Hospital (Beijing) are acute care, general hospitals serving urban populations. Case identification, laboratory diagnosis and management procedures at the individual study sites have been described previously [5, 6, 9-12]. Influenza cases were prospectively identified and diagnosed; patients presenting with symptoms of acute respiratory infection were admitted if they developed potentially serious medical complications (e.g. pneumonia or cardiovascular events), exacerbations of underlying conditions (e.g. chronic obstructive pulmonary disease (COPD) or asthma), or severe systemic/respiratory symptoms, which were unmanageable at home or as an outpatient. Following admission, patients were tested for influenza virus infections as part of hospital care and/or a surveillance programme. Virological diagnosis was established using reverse transcription PCR, and/or a combination of an immunofluorescence assay and virus culture. Chest radiography and bacterial culture of respiratory samples were routinely performed for patient management; blood cultures were performed when there were signs of sepsis. NAI (typically oral oseltamivir) were available for treatment at all sites and considered by the attending physicians based on clinical guidelines, which were evolving during the study period $[3,13,14]$.

Data on patient characteristics, complications, treatments and outcomes, collected prospectively and retrospectively at individual sites [5, 6, 9-12], were extracted and combined into a central database using standardised definitions for variables (see later). A total of 2665 cases were collated; 16 were subsequent excluded because they did not fulfil the specified age criteria $(\mathrm{N}=2649$ : Hong Kong $\mathrm{n}=1893$; Singapore $\mathrm{n}=408$; Beijing $\mathrm{n}=348$ ). All patient identifying information had been removed from the dataset and only anonymous data were used for analysis. This study was investigator-initiated and supported by a

Received: Sept 172014 | Accepted after revision: Nov 112014 | First published online: Jan 082015

Support statement: This project is jointly supported by the Research Fund for the Control of Infectious Diseases (grant CU-11-01-01), Food and Health Bureau of the Hong Kong Special Administrative Region Government; a departmental research fund, Faculty of Medicine, The Chinese University of Hong Kong, Hong Kong; and research grants from the National Natural Science Foundation of China (grants 81070005/H0104, 81030032/H19 and 81271840), Beijing. Funding information for this article has been deposited with FundRef.

The views expressed are those of the authors and do not reflect the official policies of the Centers for Disease Control and Prevention.

Conflict of interest: Disclosures can be found alongside the online version of this article at erj.ersjournals.com 
government research grant. Ethical approval for data collection was obtained from the institutional review boards of the participating sites.

\section{Definition of variables and study outcomes}

The primary outcome of the analysis was all-cause mortality within 30 days. The secondary outcomes were mortality within 60 days and duration of hospitalisation of survivors. "Comorbidity" was defined as the presence of one or more pre-existing, major medical conditions that are known risk factors for influenza-related complications (hypertension or hypercholesterolaemia alone were not considered high-risk conditions) (table 1) [5, 13]. "Pneumonia" was defined clinically and radiographically $[5,6]$. "Bacterial superinfection" was defined as positive culture of a bacterial pathogen from a lower respiratory tract specimen (sputum, bronchial/tracheal aspirates or bronchoalveolar lavage) and/or blood samples collected during the acute illness, excluding results from other sites (e.g. urine, stool or skin wound) [5, 6]. NAI treatment referred to the administration of oseltamivir, zanamivir or peramivir for one or more doses during the course of illness (table 1). Systemic corticosteroid use was defined as any intended therapeutic use of corticosteroids (e.g. methylprednisolone, prednisolone or hydrocortisone) via the parental or enteral routes during hospitalisation; replacement doses and inhalational treatment were excluded. Statin use referred to chronic use of a statin (for any length of time) prior to and at the time of hospitalisation.

\section{Data analysis}

Factors affecting patient survival were analysed. Chi-squared tests with continuity correction, Kaplan-Meier analysis and the log-rank test were used for univariate comparisons. Independent factors were determined using multivariate Cox regression models; age, sex, type/subtype of virus, NAI use, NAI treatment propensity score, bacterial superinfection, and systemic corticosteroid and statin use (all variables significant in the univariate comparisons) were included as covariates (model 1) [3, 5, 7, 8, 13]. The adjusted hazard ratios (HR) of the explanatory variables and their 95\% confidence intervals were calculated; a $\mathrm{HR}<1$ indicated a lower probability of death. To investigate whether time of treatment initiation would affect survival, NAI use was further categorised into those who started treatment within $0-2,3-5$ and $>5$ days from illness onset (model 2) $[3,5,7]$. In an attempt to address possible immortal time bias on treatment effectiveness, we also performed time-dependent Cox regressions with NAI initiation modelled as a time-dependent covariate (with respect to the day of illness onset) [15, 16], with adjustment for the same variables (model 3). Furthermore, analysis was performed among patients with "pneumonia", a defined, severe form of influenza complication (model 4) [5]. The propensity score for the likelihood of an individual patient to receive NAI treatment was calculated by a multivariate logistic regression method using the following variables [16, 17]: 1) comorbidity (defined earlier); 2) requirement for assisted ventilation and/ or intensive care unit (ICU) admission (illness severity); 3) admission year (reflecting guideline changes preand post-pandemic); and 4) study site (possible differences in clinical practice) $[3,13]$. Survival at 30 days and 60 days was analysed, because late deaths might occur due to complicated courses of illness and/or decompensation of the underlying conditions [6].

Factors affecting duration of hospitalisation of survivors were analysed using multivariate linear models, $\beta$-coefficients and $95 \%$ confidence intervals were calculated to provide adjusted estimates of differences in length of stay between the comparing factors. A subanalysis of patients who had respiratory failure, defined as hypoxaemia requiring supplemental oxygen support (i.e. among patients with more severe diseases) [5, 6], was performed.

Cox regressions and multivariate linear models were performed using the Proc PHREG and Proc GLM procedures in SAS, respectively (SAS version 9.3; SAS Institue Inc., Cary, NC, USA). Descriptive and univariate analyses were performed using the PASW Statistics version 18.0 (SPSS Inc., Chicago, IL, USA). All probabilities were two-tailed and a $\mathrm{p}$-value of $<0.05$ was considered to indicate statistical significance.

\section{Results}

Descriptions of cohort

Altogether data for 2649 hospitalised patients with laboratory-confirmed influenza were analysed. Their baseline characteristics, virological diagnoses, complications, treatments received and clinical outcomes are described in table 1. The numbers of patients enrolled in 2008, 2009, 2010 and 2011 were 516 (19.5\%), 1008 (38.1\%), 660 (24.9\%) and 465 (17.5\%), respectively, including 2354 cases of influenza A and 295 cases influenza B (seasonal influenza A/B viruses 56.9\%, influenza A(H1N1)pdm09 virus 36.3\%). Lower respiratory tract specimens were used to establish the diagnosis in $9.1 \%$ of cases. The median age of the cohort was 63 years (interquartile range (IQR) $42-79$ years) and $60.6 \%$ had one or more major comorbidities. The median time interval between illness onset and presentation to the hospitals was 2 days (IQR 1-3 days). Defined pulmonary and/or extrapulmonary complications were present in $68.4 \%$ of cases; clinicoradiological pneumonia was diagnosed in $40.8 \%$. Culture-proven bacterial superinfections occurred 
TABLE 1 Characteristics, complications, treatments and outcomes of 2649 adults hospitalised with virologically confirmed influenza virus infections

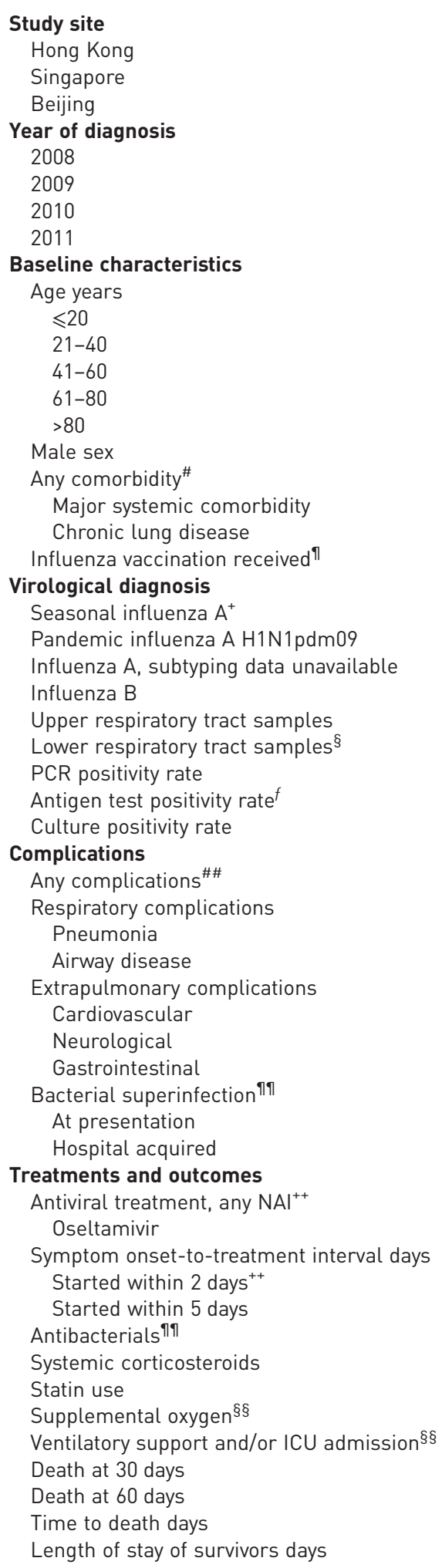

$1893(71.5)$

$408(15.4)$

$348(13.1)$

$516(19.5)$

$1008(38.1)$

$660(24.9)$

465 (17.5)

$63.0(42.0-79.0)$

102 (3.9)

525 (19.8)

$606(22.9)$

$833(31.4)$

$583(22.0)$

$1370(51.7)$

$1604(60.6)$

$1318(49.8)$

$561(21.2)$

$207(17.5)$

$1212(45.8)$

961 (36.3)

181 (6.8)

295 (11.1)

2131 (90.8)

213 (9.1)

$1335 / 1465$ (91.1)

1528/1901 (80.4)

$1543 / 1793(86.1)$

1811 (68.4)

1479 (55.8)

1081 (40.8)

510 (19.3)

410 (15.5)

193 (7.3)

109 (4.1)

134 (5.1)

286 (10.8)

185 (7.0)

114 (4.3)

1991 (75.2)

1956 (73.8)

$2(1-4)$

$1160(44.5)$

1706 (65.5)

2282 (88.1)

$610(23.1)$

336 (12.7)

$1249(48.6)$

305 (11.5)

156 (5.9)

183 (6.9)

8 (3-17)

5 (3-9)

Data are presented as $\mathrm{n}(\%), \mathrm{n} / \mathrm{N}(\%)$ or median (interquartile range), unless otherwise stated. Other patient characteristics: ethnic Chinese 92.8\%; nursing home residents 13.9\% (344 out of 2478); obesity 2.2\% (49 out of 2253); pregnant women $10.7 \%$ (44 out of 411 reproductive age females). NAl: neuraminidase 


\section{TABLE 1 Continued}

inhibitors; ICU: intensive care unit. \#: major systemic comorbidity [4, 12] defined as congestive heart failure, cerebrovascular, neoplastic, or chronic liver or renal diseases, diabetes mellitus, chronic cardiovascular, autoimmune or neurological diseases, or immunosuppression Inot hypertension or hypercholesterolaemia alone); chronic lung diseases were defined as chronic obstructive pulmonary disease (COPD), asthma, bronchiectasis and pulmonary fibrosis. ף: data on influenza vaccine status available in 1181 patients only. ${ }^{+}$: most cases were influenza $\mathrm{A} / \mathrm{H} 3 \mathrm{~N} 2$; only 150 seasonal influenza $\mathrm{A} / \mathrm{H} 1 \mathrm{~N} 1$ cases were confirmed during 2008 and early 2009, of which 73 did not receive oseltamivir treatment; the remainder received either zanamivir, or oseltamivir plus amantadine. §. diagnostic specimen data were available in 2346 cases; lower respiratory tract samples included sputum, endotracheal aspirates and bronchoalveolar lavage, as diagnostic specimens, upper respiratory samples included nasopharyngeal aspirates and nasal/throat swabs. ${ }^{f}$ : antigen tests (Hong Kong only); 1784 out of 1901 (93.8\%) were immunofluorescence assay, positivity rate for $\mathrm{H} 1 \mathrm{~N} 1 \mathrm{pdm09}$ virus was $64.2 \%$. \#\# complications [4, 5, 12] were categorised as respiratory (clinicoradiographical pneumonia, acute bronchitis, acute exacerbations of asthma or COPD), extrapulmonary (cardiovascular (e.g. acute coronary syndrome, decompensated heart failure, arrhythmia or myocarditis), neurological (e.g. encephalopathy, confusion or delirium, seizure, stroke or transient ischaemic attack), gastrointestinal (e.g. severe vomiting, diarrhoea, abdominal pain, gastrointestinal bleeding or liver derangement)) or other (metabolic derangement, renal failure, sepsis syndrome, syncope, etc.). ๆๆ: bacterial superinfection $[4,5]$ was evidenced by positive culture of a bacterial pathogen from respiratory (sputum or bronchial/tracheal aspirates) and/or blood samples; infections with onset > 2 days after admission were considered hospital acquired (concurrent Aspergillus infections, $\mathrm{n}=7$ ); antibacterial data unknown $(n=121) .{ }^{++}$: antiviral treatment included oseltamivir use $(n=1956)$, inhalational zanamivir alone $(n=30)$, peramivir alone $(n=5)$, oseltamivir and peramivir $(n=5)$, oseltamivir and zanamivir $(n=18)$, oseltamivir and amantadine $(n=35)$, no antiviral $(n=658)$; symptom onset date unknown ( $n=44)$, treatment duration $>5$ days (21.6\%). $\S \S$ : data on supplemental oxygen use unknown ( $n=78)$; ventilatory support included invasive or noninvasive ventilatory support.

in $10.8 \%$ of cases, of which $4.3 \%$ were hospital-acquired (table 1 ). About half $(48.6 \%)$ of the patients had respiratory failure requiring supplemental oxygen support; invasive/noninvasive ventilation or ICU admission was required for life-support in $11.5 \%$. The crude all-cause mortality at 30 and 60 days was $5.9 \%(\mathrm{n}=156)$ and $6.9 \%(\mathrm{n}=183)$, respectively (mortality in NAI-treated versus - untreated was $5.3 \%$ versus

TABLE 2 Factors associated with death at 30 and 60 days

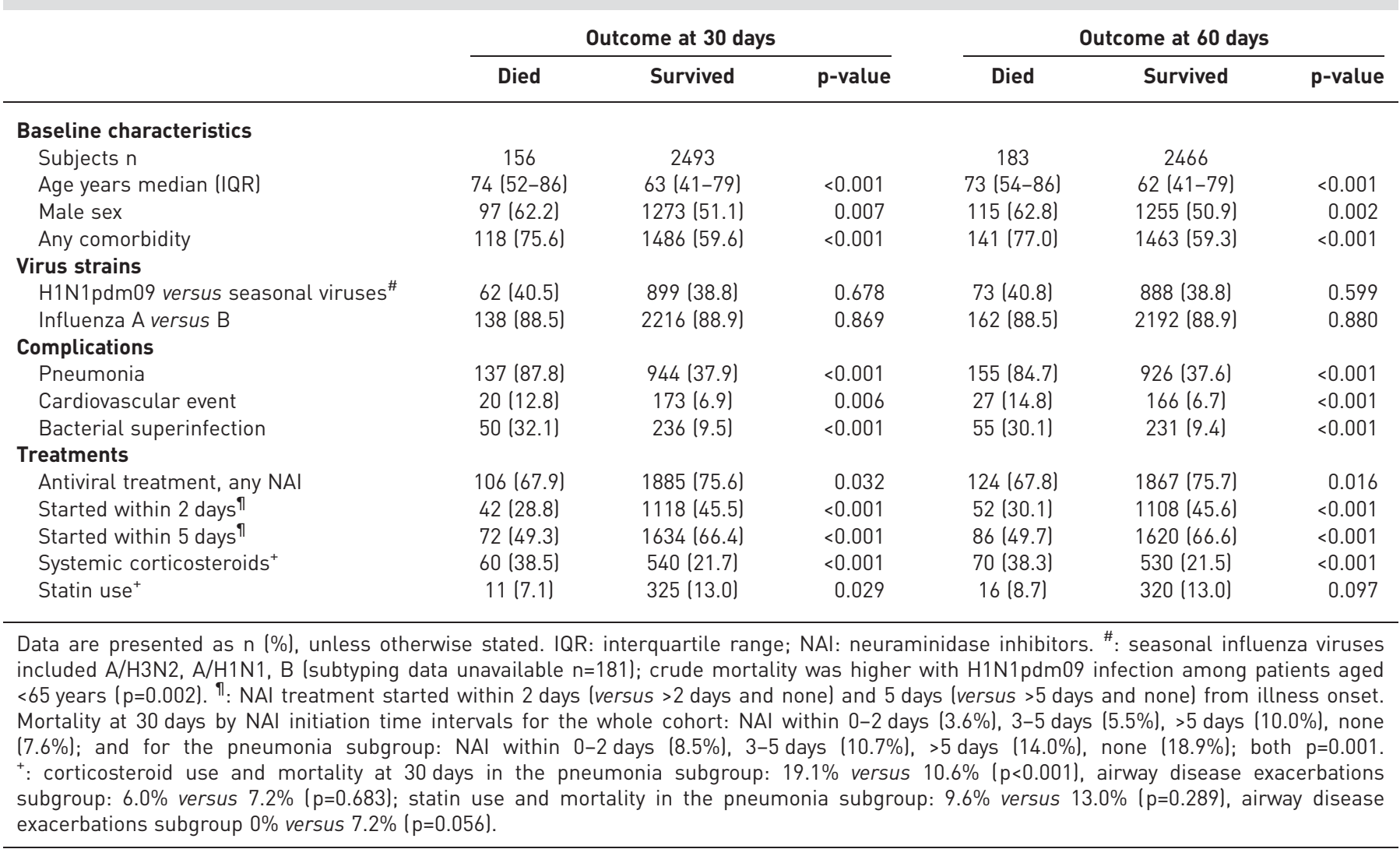


$7.6 \%$ and $6.2 \%$ versus $9.0 \%$, at 30 and 60 days respectively) (table 2). Cumulative proportions of death at 30, 40 and 50 days were $85 \%, 90 \%$ and 95\%, respectively. For the survivors, the median hospital length-of-stay was 5 days (IQR 3-9 days).

Overall, 1991 (75.2\%) patients received NAI treatment, of whom 1956 (73.8\%) were treated with either oseltamivir or an oseltamivir-containing regimen (other NAI: zanamivir or peramivir alone, $\mathrm{n}=35$ ) (table 1). Proportions of NAI-treated patients in 2008, 2009, 2010 and 2011 were $29.7 \%, 88.9 \%$, 88.9\% and 76.3\%, respectively. Treatment was initiated within $0-2$ days of illness in $44.5 \%, 3-5$ days in $21.0 \%$ and $>5$ days in 9.3\%. Systemic corticosteroids were given in 23.1\%; a higher proportion of bacterial superinfection during the course of hospitalisation was found among the corticosteroid recipients $(9.7 \%$ versus $2.7 \%$; $\mathrm{p}<0.001)$. There were 336 chronic statin users in this cohort; compared with the nonusers, they were significantly older $(73 \pm 11$ versus $58 \pm 23$ years; $\mathrm{p}<0.001)$ and major comorbidities were more frequently present $(94.3 \%$ versus $55.6 \%, \mathrm{p}<0.001)$. Proportions with defined complications were similar $(70.8 \%$ versus $68.0 \% ; \mathrm{p}=0.298)$.

\section{Factors associated with mortality}

In univariate analyses (table 2), older age, male sex, comorbidities, pneumonia, bacterial superinfections $(17.5 \%$ versus $4.5 \%)$ and corticosteroid use $(10.0 \%$ versus $4.7 \%)$ were significantly associated with mortality. Patients who received NAI treatment within 2 days of illness or within 5 days of illness, and those who were on chronic statin therapy (3.3\% versus $6.3 \%$ ) were shown to have lower risks of death at 30 days. Results were similar for death at 60 days. Kaplan-Meier curves comparing survival with regard to NAI treatment status, treatment initiation time, corticosteroids and statin use are shown in figure 1a-d.
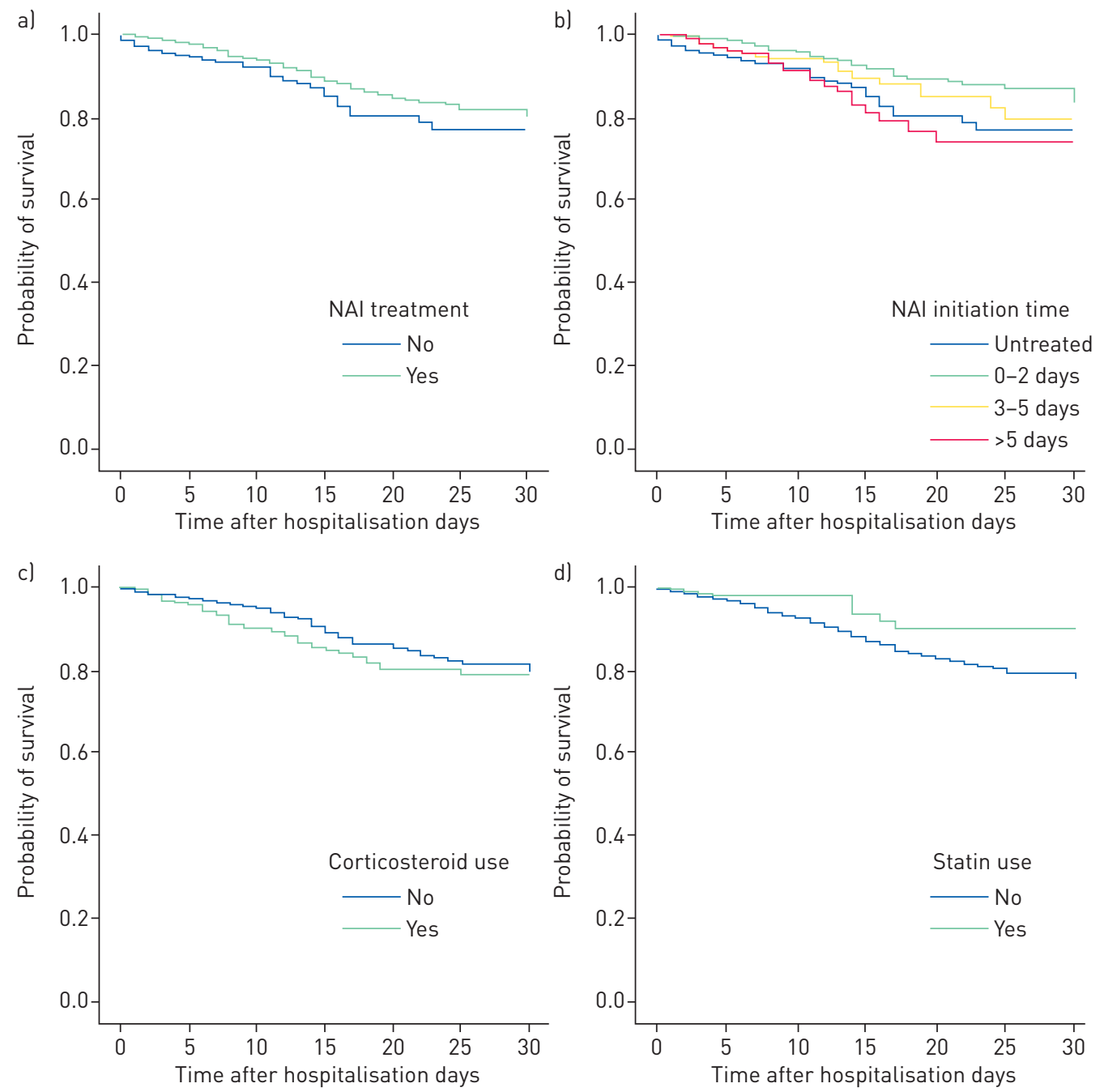

FIGURE 1 Kaplan-Meier survival curves of adult patients hospitalised for confirmed influenza virus infections, censored at 30 days. Survival according to a) neuraminidase inhibitors (NAI) treatment status (log-rank test $\mathrm{p}=0.002$ ), b) NAI initiation time (log-rank test $\mathrm{p}<0.001)$, c) systemic steroid use (log-rank test $\mathrm{p}=0.038$ ) and d) chronic statin use (log-rank test $\mathrm{p}=0.013$ ). 
Results of multivariate Cox regression analyses (censored at 30 days) are shown in table 3 . In model 1, NAI treatment was found to be an independent factor associated with improved survival versus no treatment (adjusted HR 0.28, 95\% CI 0.19-0.43; p<0.001), adjusted for propensity score, patient characteristics and virus type/subtype. Bacterial superinfection (adjusted HR 2.18, 95\% CI 1.52-3.11; $\mathrm{p}<0.001$ ) and chronic statin use (adjusted HR 0.44, 95\% CI 0.23-0.84; $\mathrm{p}=0.013$ ) were associated with increased and decreased death risks, respectively. In model 2, early NAI treatment within 2 days of illness was associated with the highest chance of survival (adjusted HR 0.20, 95\% CI 0.12-0.32; $\mathrm{p}<0.001$ ), but improved survival was also observed with treatment started within 3-5 days, compared with no treatment (adjusted HR 0.35, 95\% CI 0.21-0.58; p<0.001). In model 3, NAI initiation, modelled as a time-dependent covariate, was significantly associated with survival (adjusted HR 0.39 , 95\% CI 0.27-0.57; $\mathrm{p}<0.001$ ), adjusted for the same confounders. Model 4, the analysis of complicated infections with pneumonia, showed similar results. The progressive increase in the hazard ratios with each day of delay in starting NAI treatment after illness onset is shown in supplementary figure S1.

\section{TABLE 3 Adjusted hazard ratios of explanatory variables associated with death, as shown in} multivariate Cox regression models (censored at 30 days)

Adjusted hazard ratio $(95 \% \mathrm{CI})$

p-value

\section{Model $1^{\#}$}

Age per 20 years

Male sex

H1N1pdm09

Bacterial superinfection

NAl treatment

Statin use

Systemic corticosteroids

\section{Model 2"}

Age per 20 years

Male sex

H1N1pdm09

Bacterial superinfection

NAl treatment

$0-2$ days

$3-5$ days

$>5$ days

None

Statin use

Systemic corticosteroids

\section{Model $3^{+}$}

Age per 20 years

Male sex

H1N1pdm09

Bacterial superinfection

NAl treatment ${ }^{\S}$

Statin use

Systemic corticosteroids

\section{Model $4^{f}$}

Age per 20 years

Male sex

H1N1pdm09

Bacterial superinfection

NAl treatment ${ }^{\S}$

Statin use

Systemic corticosteroids

$\begin{array}{lr}1.13(0.95-1.36) & 0.170 \\ 1.38(1.00-1.92) & 0.053 \\ 1.35(0.90-2.02) & 0.142 \\ 2.18(1.52-3.11) & <0.001 \\ 0.28(0.19-0.43) & <0.001 \\ 0.44(0.23-0.84) & 0.013 \\ 1.11(0.79-1.56) & 0.554 \\ & \\ 1.21(1.00-1.46) & 0.050 \\ 1.48(1.05-2.09) & 0.024 \\ 1.15(0.74-1.79) & 0.538 \\ 2.14(1.48-3.10) & <0.001 \\ & \\ 0.20(0.12-0.32) & <0.001 \\ 0.35(0.21-0.58) & <0.001 \\ 0.49(0.27-0.88) & 0.017 \\ & \\ 0.49(0.26-0.94) & 0.032 \\ 1.04(0.73-1.48) & 0.839 \\ 1.15(0.95-1.38) & \\ 1.34(0.96-1.86) & 0.148 \\ 1.11(0.75-1.65) & 0.083 \\ 2.18(1.53-3.11) & 0.611 \\ 0.39(0.27-0.57) & <0.001 \\ 0.42(0.22-0.81) & <0.001 \\ 1.09(0.77-1.53) & 0.009 \\ 1.01(0.82-1.24) & 0.629 \\ 1.16(0.82-1.65) & \\ 0.65(0.42-1.02) & 0.935 \\ 1.50(1.03-2.19) & 0.392 \\ 0.39(0.26-0.59) & 0.062 \\ 0.59(0.30-1.13) & 0.034 \\ 1.12(0.78-1.61) & 0.553\end{array}$

In all models, neuraminidase inhibitors (NAl) treatment propensity score was included as a covariate (see methods), and had a $p$-value $<0.05$. An adjusted hazard ratio $<1$ indicated a lower probability of death. \#: model 1 was adjusted for NAl treatment, bacterial superinfection, statin and corticosteroid use lyes versus nol and H1N1pdm09 (versus seasonal influenza A/B viruses). ": model 2 was adjusted for NAl treatment; "none" was the reference group. ${ }^{+}$: time-dependent Cox regression. ${ }^{\S}$ : models 3 and 4 were time-dependent Cox regressions; NAl treatment initiation with respect to day of illness onset was analysed as a time-dependent covariate (versus none). ${ }^{f}$ : pneumonia subgroup. 
Survival analyses censored at 60 days showed consistent results; NAI treatment, bacterial superinfection and statin use were the significant variables (supplementary table S1). We further examined the impact of systemic corticosteroid use in a post hoc analysis excluding patients with known indications for the treatment of acute airway disease exacerbations. Corticosteroid administration was shown to be significantly associated with increased death risks (adjusted HR 1.73, 95\% CI 1.14-2.62; $\mathrm{p}=0.010$ ) (supplementary table S2 and table 2).

\section{Factors associated with duration of hospitalisation}

Analyses of duration of hospitalisation data are shown in table 4. For all survivors, it was found that older age, bacterial superinfection and corticosteroid use were significantly associated with length of stay, whereas an insignificant trend of association was shown for NAI treatment. In a subanalysis of patients with respiratory failure, however, significantly longer length of stay was observed in patients who did not receive NAI treatment within 5 days of illness $(p=0.023)$. Those who received early treatment within 2 days of illness had the shortest length of stay (table 4 ).

\section{Discussion}

In this large, multicentre cohort study, we show that adults hospitalised for seasonal or pandemic influenza have high morbidity and mortality. Apart from the conventional risk factors such as old age and comorbidity, we found that NAI treatment, secondary infections, systemic corticosteroid and statin use may affect clinical outcomes. These findings have important implications for patient care.

Our results are consistent with other cohort studies and recent meta-analyses which showed that early NAI treatment is associated with improved survival in $\mathrm{H} 1 \mathrm{~N} 1 \mathrm{pdm} 09$ influenza patients $[7,16]$. In this analysis, we included both seasonal and pandemic virus subtypes, and carefully controlled for important confounders including patient characteristics, disease severity, propensity to receive NAI treatment and possible immortal time bias, with the aim of providing a more accurate estimate on the effectiveness of antiviral intervention on outcomes. Effects on survival, as well as illness duration, were studied. Overall, NAI treatment was found to be associated with a reduced risk of death by $\sim 72 \%$ (adjusted HR $0.28,95 \%$ CI $0.19-0.43$ ) in the time-fixed, and 61\% (adjusted HR 0.39, 95\% CI 0.27-0.57) in the time-dependent analyses; significant results were also observed in cases complicated by pneumonia (table 3 ). Notably, the majority (58\%) of our treated patients had received NAI within 2 days of illness and they were shown to have the best chance of survival. Benefits were also found with later treatment initiation, but the magnitude was smaller, and decreased rapidly with longer time delays (fig. 1b and supplementary fig S1). In hospitalised influenza patients, a greater therapeutic time window is expected as viral replication is

TABLE 4 Independent factors associated with hospital length of stay, as shown in multivariate linear models

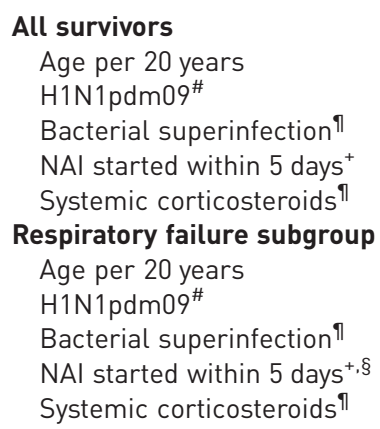

$$
\begin{gathered}
1.72(1.30-2.14) \\
2.20(1.28-3.11) \\
7.63(6.31-8.94) \\
-0.74(-1.58-0.10) \\
3.15(2.19-4.10) \\
1.56(0.62-2.50) \\
1.75(-0.07-3.56) \\
9.83(7.73-11.94) \\
-1.97(-3.68--0.27) \\
1.54(-0.02-3.11)
\end{gathered}
$$

$<0.001$

$<0.001$

$<0.001$

Data are presented as adjusted estimates $(\beta)$ and $95 \%$ confidence intervals of the differences in length of stay (LOS) (days). Comorbidity, sex and statin use were adjusted in all models, which showed insignificant results. NAl: neuraminidase inhibitors. \#: H1N1pdm09 versus seasonal influenza A/B viruses. ": yes versus no. ${ }^{+}$: NAl treatment started within 5 days of illness versus no or later treatment. ${ }^{\S}$ : estimated differences in LOS ( $\beta$ ) with NAl started within $0-2$ days versus none $(\beta-2.08,95 \% \mathrm{Cl}-4.36-0.20), 3-5$ days versus none $(\beta-1.08,95 \% \mathrm{Cl}-3.71-1.54)$ and $>5$ days versus none $(\beta 0.50,95 \% \mathrm{Cl}-2.59-3.60)$. Respiratory failure was present at the time of NAl initiation in nearly all cases. A supplementary analysis using the time-dependent Cox regression method showed a significant association between NAI use and hospital discharge ladjusted hazard ratio $1.22,95 \% \mathrm{Cl} 1.10-1.34, \mathrm{p}<0.001$; data not shown). 
usually more prolonged; however, if the disease has progressed to an advanced stage, antivirals alone may not reverse the course because of extensive inflammation-mediated tissue damage [8, 18-20]. Along the continuum, findings of effectiveness appear to be most consistent with treatment started within 5 days [21-24]; however, a small benefit with later treatments cannot be ruled out. We also found evidence that early NAI treatment may shorten length of stay when patients with more severe diseases were analysed (see later) $[6,18,25]$. Our data support the recommendation to start NAI as soon as possible in all patients hospitalised with confirmed or suspected influenza, including those who present after 2 days following onset [13]. We noted that although the time to presentation was $\leqslant 2$ days in $50 \%$ and $\leqslant 5$ days in $85 \%$ of cases, considerably fewer were given antivirals and, as in western countries, there was a drop in antiviral use after the 2009 pandemic [26]. Strategies to improve rapid diagnosis, accessibility and use of antivirals are urgently required $[3,4,27]$.

Bacterial superinfections were documented in $\sim 11 \%$ of cases ( $>4 \%$ hospital acquired), and were associated with a two-fold increase in death risk and an extended period of hospitalisation. The true incidence could be even higher as only culture-proven cases were reported [5]. It is increasingly recognised that complex interactions exist between the co-infecting pathogens and the host, leading to disruption of physical barriers, outgrowth of pathogens and potentiated inflammation-mediated injury [28]. As such, prompt detection of bacterial superinfection and appropriate antibiotic coverage are advisable [3, 27]. Besides the usual pathogens Streptococcus pneumoniae and Staphylococcus aureus, drug-resistant Gram-negative bacteria are a particular concern in our region $[6,12]$. Measures to prevent nosocomial infections, particularly ventilator-associated pneumonia, should be implemented [29]. We reported more superinfections (9.7\% versus 2.7\%) and deaths ( $10.0 \%$ versus $4.7 \%$ ) among patients receiving systemic corticosteroids, the death risk was significant (adjusted HR 1.7, 95\% CI 1.1-2.6) after controlling for indications and confounders (table 2 and supplementary table S2) [30-32]. Available evidence indicates that corticosteroids do not effectively suppress inflammation in influenza but further impair host defence resulting in delayed viral clearance and bacterial/ fungal sepsis [8, 18, 27, 30-32]. Although selection bias ("treating the sickest") cannot be eliminated, our results suggest that corticosteroids should not be administered routinely for the treatment of influenza pneumonia. In patients with established indications, such as therapy for acute airway disease exacerbations, it may be prudent to monitor for viral clearance and minimise corticosteroid exposure $[8,18,27]$.

Numerous reports have described benefits of statin use in sepsis, pneumonia and COPD exacerbation, which have been attributed to their anti-inflammatory properties $[8,33,34]$. Data on influenza are limited, and recently, an association with lower mortality risk (adjusted for vaccination status) in hospitalised influenza patients has been reported [33,35]. We observed fewer deaths among the chronic statin users (3.3\% versus $6.3 \%$ ), despite their older age and the presence of major comorbidities (not including hypercholesterolaemia), which is not readily explainable by the "healthy user bias" [36]. Recent trials on acute statin administration in non-influenza diseases (e.g. sepsis or acute respiratory distress syndrome) have shown negative results [37], but continuation of pre-existing therapy seems beneficial [38]. Given these observations, further studies on the immunological/metabolic effects of prior, chronic statin use in influenza patients is still warranted, which may provide insights into pathogenesis and adjunctive therapy research $[8,33,36,38,39]$.

The strengths of our study include a large sample size, multicentre design and virological confirmation of cases. Important confounders, including the propensity to receive antiviral treatment, have been carefully controlled. Results from univariate and multivariate analyses are consistent, and both time-fixed and time-dependent regressions were performed to provide estimates of risks [40]. Limitations pertaining to observational studies are unavoidable but it is unlikely that selection bias, such as omitting antivirals for milder infections, could explain the mortality differences. Early discharge of mild, untreated patients is possible; therefore, treatment impact on length of stay was further examined among the severe cases. Since only a small proportion of cases $(<6 \%)$ was infected with the seasonal $\mathrm{A}(\mathrm{H} 1 \mathrm{~N} 1)$ virus $(\sim 60 \%$ oseltamivir resistant in $2008-$ 2009) $[6,41]$, and most had either received zanamivir or remained untreated (table 1), the conclusions should be unaffected. We are unable to study the impact of influenza vaccination due to incomplete data, but vaccination coverage in Asian populations is known to be very low and the monovalent A(H1N1)pdm09 vaccine was largely unavailable during the 2009 pandemic [5, 6, 9, 11, 42, 43]. Given the reported high prevalence of the IFITM3 CC-genotype (associated with influenza progression) in Asian populations [44], future studies may need to consider genetic/ethnicity factors when analysing disease outcomes.

In conclusion, our data suggest that NAI treatment may improve survival of hospitalised influenza patients. The benefit is greatest with, but not limited to, treatment started within 2 days of illness. Secondary infections and corticosteroids may increase death risks. In view of these findings, the overall management approach will need to consider both antiviral (e.g. early diagnosis and treatment, and empirical treatment of suspect cases) and non-antiviral (e.g. treatment and prevention of superinfections, avoidance of corticosteroids or other immunosuppressants) strategies to optimise patient outcomes. 


\section{References}

1 World Health Organization (WHO). Influenza. www.who.int/influenza/en/ Date last accessed: September 1, 2014. Leo YS, Lye DC, Chow A. Influenza in the tropics. Lancet Infect Dis 2009; 9: 457-458.

Lee N, Ison MG. Diagnosis, management and outcomes of adults hospitalized with influenza. Antivir Ther 2012; 17: $143-157$.

4 Dawood FS, Iuliano AD, Reed C, et al. Estimated global mortality associated with the first 12 months of 2009 pandemic influenza A H1N1 virus circulation: a modelling study. Lancet Infect Dis 2012; 12: 687-695.

5 Lee N, Chan PK, Lui GC, et al. Complications and outcomes of pandemic 2009 influenza A (H1N1) virus infection in hospitalized adults: how do they differ from those in seasonal influenza? J Infect Dis 2011; 203: 1739-1747.

6 Lee N, Choi KW, Chan PK, et al. Outcomes of adults hospitalised with severe influenza. Thorax 2010; 65: 510-515.

7 Hsu J, Santesso N, Mustafa R, et al. Antivirals for treatment of influenza: a systematic review and meta-analysis of observational studies. Ann Intern Med 2012; 156: 512-524.

8 Hui DS, Lee N, Chan PK. Adjunctive therapies and immunomodulatory agents in the management of severe influenza. Antiviral Res 2013; 98: 410-416.

9 Leo YS, Lye DC, Barkham T, et al. Pandemic (H1N1) 2009 surveillance and prevalence of seasonal influenza, Singapore. Emerg Infect Dis 2010; 16: 103-105.

10 Win MK, Chen MI, Barkham T, et al. Influenza disease burden in adults by subtypes following the initial epidemic of pandemic H1N1 in Singapore. Influenza Other Respir Viruses 2011; 5: e563-e567.

11 Yang SQ, Qu JX, Wang C, et al. Influenza pneumonia among adolescents and adults: a concurrent comparison between influenza A (H1N1) pdm09 and A (H3N2) in the post-pandemic period. Clin Respir J 2014; 8: 185-191.

12 Bai L, Gu L, Cao B, et al. Clinical features of pneumonia caused by 2009 influenza A(H1N1) virus in Beijing, China. Chest 2011; 139: 1156-1164.

13 Fiore AE, Fry A, Shay D, et al. Antiviral agents for the treatment and chemoprophylaxis of influenza recommendations of the Advisory Committee on Immunization Practices (ACIP). MMWR Recomm Rep 2011; 60: $1-24$.

14 Zhong NS, Li YM, Yang ZF, et al. Chinese guidelines for diagnosis and treatment of influenza (2011). $J$ Thorac Dis 2011; 3: 274-289.

$15 \mathrm{Xu} \mathrm{R}$, Luo Y, Chambers C. Assessing the effect of vaccine on spontaneous abortion using time-dependent covariates Cox models. Pharmacoepidemiol Drug Saf 2012; 21: 844-850.

16 Muthuri SG, Venkatesan S, Myles PR, et al. Effectiveness of neuraminidase inhibitors in reducing mortality in patients admitted to hospital with influenza A H1N1pdm09 virus infection: a meta-analysis of individual participant data. Lancet Respir Med 2014; 2: 395-404.

17 Adisasmito W, Chan PK, Lee N, et al. Effectiveness of antiviral treatment in human influenza A (H5N1) infections: analysis of a Global Patient Registry. J Infect Dis 2010; 202: 1154-1160.

18 Lee N, Chan PK, Hui DS, et al. Viral loads and duration of viral shedding in adult patients hospitalized with influenza. J Infect Dis 2009; 200: 492-500.

19 Ling LM, Chow AL, Lye DC, et al. Effects of early oseltamivir therapy on viral shedding in 2009 pandemic influenza A (H1N1) virus infection. Clin Infect Dis 2010; 50: 963-969.

20 Chan PK, Lee N, Zaman M, et al. Determinants of antiviral effectiveness in influenza virus A subtype H5N1. J Infect Dis 2012; 206: 1359-1366.

21 Louie JK, Yang S, Acosta M, et al. Treatment with neuraminidase inhibitors for critically ill patients with influenza A (H1N1)pdm09. Clin Infect Dis 2012; 55: 1198-1204.

22 Yu H, Feng Z, Uyeki TM, et al. Risk factors for severe illness with 2009 pandemic influenza A (H1N1) virus infection in China. Clin Infect Dis 2011; 52: 457-465.

23 Fry AM, Goswami D, Nahar K, et al. Efficacy of oseltamivir treatment started within 5 days of symptom onset to reduce influenza illness duration and virus shedding in an urban setting in Bangladesh: a randomised placebo-controlled trial. Lancet Infect Dis 2014; 14: 109-118.

24 Yang SG, Cao B, Liang LR, et al. Antiviral therapy and outcomes of patients with pneumonia caused by influenza A pandemic (H1N1) virus. PLoS One 2012; 7: e29652.

25 Higuera Iglesias AL, Kudo K, Manabe T, et al. Reducing occurrence and severity of pneumonia due to pandemic H1N1 2009 by early oseltamivir administration: a retrospective study in Mexico. PLoS One 2011; 6: e21838.

26 Garg S, Chaves SS, Pérez A, et al. Reduced influenza antiviral treatment among children and adults hospitalized with laboratory-confirmed influenza infection in the year after the 2009 pandemic. Clin Infect Dis 2012; 55: e18-e21.

27 Uyeki TM. Preventing and controlling influenza with available interventions. N Engl J Med 2014; 370: 789-791.

28 McCullers JA. The co-pathogenesis of influenza viruses with bacteria in the lung. Nat Rev Microbiol 2014; 12: 252-262.

29 Rebmann T, Greene LR. Preventing ventilator-associated pneumonia: an executive summary of the Association for Professionals in Infection Control and Epidemiology, Inc, Elimination Guide. Am J Infect Control 2010; 38: 647-649.

30 Kim SH, Hong SB, Yun SC, et al. Corticosteroid treatment in critically ill patients with pandemic influenza A/H1N1 2009 infection: analytic strategy using propensity scores. Am J Respir Crit Care Med 2011; 183: $1207-1214$.

31 Han K, Ma H, An X, et al. Early use of glucocorticoids was a risk factor for critical disease and death from pH1N1 infection. Clin Infect Dis 2011; 53: 326-333.

32 Lee N, Hui DS. Dexamethasone in community-acquired pneumonia. Lancet 2011; 378: 979-980.

33 Fedson DS. Treating influenza with statins and other immunomodulatory agents. Antiviral Res 2013; 99: 417-435.

34 Mortensen EM, Nakashima B, Cornell J, et al. Population-based study of statins, angiotensin II receptor blockers, and angiotensin-converting enzyme inhibitors on pneumonia-related outcomes. Clin Infect Dis 2012; 55: 1466-1473.

35 Vandermeer ML, Thomas AR, Kamimoto L, et al. Association between use of statins and mortality among patients hospitalized with laboratory-confirmed influenza virus infections: a multistate study. J Infect Dis 2012; 205: 13-19. 
Walsh EE. Statins and influenza: can we move forward? J Infect Dis 2012; 205: 1-3.

37 The National Heart, Lung, and Blood Institute ARDS Clinical Trials Network, Truwit JD, Bernard GR, et al. Rosuvastatin for sepsis-associated acute respiratory distress syndrome. N Engl J Med 2014; 370: 2191-2200.

38 Kruger P, Bailey M, Bellomo R, et al. A multicenter randomized trial of atorvastatin therapy in intensive care patients with severe sepsis. Am J Respir Crit Care Med 2013; 187: 743-750.

39 Belser JA, Szretter KJ, Katz JM, et al. Simvastatin and oseltamivir combination therapy does not improve the effectiveness of oseltamivir alone following highly pathogenic avian H5N1 influenza virus infection in mice. Virology 2013; 439: 42-46.

40 Leonardi-Bee J, Venkatesan S, Muthuri SG, et al. Statistical and methodological concerns about the beneficial effect of neuraminidase inhibitors on mortality. Lancet Respir Med 2014; 2: e10-e12.

41 Chan MC, Lee N, Lui GC, et al. Comparisons of oseltamivir-resistant (H275Y) and concurrent oseltamivir-susceptible seasonal influenza A(H1N1) virus infections in hospitalized adults, 2008-2009. Influenza Other Respir Viruses 2013; 7: 235-239.

42 Wu S, Yang P, Li H, et al. Influenza vaccination coverage rates among adults before and after the 2009 influenza pandemic and the reasons for non-vaccination in Beijing, China: a cross-sectional study. BMC Public Health 2013; 13: 636.

43 Chor JS, Ngai KL, Goggins WB, et al. Willingness of Hong Kong healthcare workers to accept pre-pandemic influenza vaccination at different WHO alert levels: two questionnaire surveys. BMJ 2009; 339: b3391.

44 Zhang $\mathrm{YH}$, Zhao $\mathrm{Y}, \mathrm{Li} \mathrm{N}$, et al. Interferon-induced transmembrane protein-3 genetic variant rs12252-C is associated with severe influenza in Chinese individuals. Nat Commun 2013; 4: 1418. 